\title{
Directed Flow in Microscopic Models in Relativistic A+A Collisions ${ }^{\dagger}$
}

\author{
Larissa Bravina $^{1, *}$, Yurii Kvasiuk ${ }^{2}$, Sergey Sivoklokov ${ }^{3}$, Oleksandr Vitiuk ${ }^{2}$ and \\ Evgeny Zabrodin ${ }^{1,3}$ \\ 1 Department of Physics, University of Oslo, PB 1048 Blindern, N-0316 Oslo, Norway; \\ eugen.zabrodin@fys.uio.no \\ 2 Taras Shevchenko National University of Kyiv, UA-01033 Kyiv, Ukraine; yurii.kvasiuk@gmail.com (Y.K.); \\ oleksandr.vitiuk@fys.uio.no (O.V.) \\ 3 Skobeltsyn Institute of Nuclear Physics, Moscow State University, Moscow RU-119991, Russia; \\ Serguei.Sivoklokov@cern.ch \\ * Correspondence: larissa.bravina@fys.uio.no \\ $+\quad$ This paper is based on the talk at the 7th International Conference on New Frontiers in Physics \\ (ICNFP 2018), Crete, Greece, 4-12 July 2018.
}

Received: 24 January 2019; Accepted: 28 February 2019; Published: 5 March 2019

\begin{abstract}
Evolution of directed flow of charged particles produced in relativistic heavy-ion collisions at energies $4 \leq \sqrt{s} \leq 19.6 \mathrm{GeV}$ is considered within two microscopic transport models, ultra-relativistic quantum molecular dynamics (UrQMD) and quark-gluon string model (QGSM). In both models, the directed flow of protons changes its sign at midrapidity from antiflow to normal flow with decreasing energy of collisions, whereas the flows of mesons and antiprotons remain antiflow-oriented. For lighter colliding systems, such as $\mathrm{Cu}+\mathrm{Cu}$ or $\mathrm{S}+\mathrm{S}$, changing of the proton directed flow occurs at lower bombarding energies and for more central topologies compared to a heavy $\mathrm{Au}+\mathrm{Au}$ system. The differences can be explained by dissimilar production zones of different hadrons and by the influence of spectators. Directed flows of most abundant hadronic species at midrapidity are found to be formed within $t=10-12 \mathrm{fm} / \mathrm{c}$ after the beginning of nuclear collision. The influence of hard and soft mean-field potentials on the directed flow is also studied.
\end{abstract}

Keywords: relativistic heavy-ion collisions; microscopic transport models; directed flow; mean-field potentials

\section{Introduction}

The collective flow was proposed as a measure of expansion of hadrons, produced in relativistic heavy-ion collisions, in both longitudinal and transverse directions in [1,2]. The first experimental measurement of the flow was done by the Plastic Ball collaboration [3] at Bevalac. Since then, the intensive study of this phenomenon by theoreticians and the experimentalists has begun. Initially, the collective flow in transverse plane, which is orthogonal to the beam axis, was decomposed onto the bounce-off flow projected on the impact parameter axis and the squeeze-out flow orthogonal to the reaction plane, see e.g., [4].

The method of decomposition of the transverse flow in infinite Fourier series was proposed in $[5,6]$. It states that the invariant cross section can be written as

$$
\begin{aligned}
E \frac{d^{3} N}{d^{3} p} & =\frac{d^{2} N}{\pi d p_{T}^{2} d y}\left\{1+2 \sum_{n=1}^{\infty} v_{n} \cos \left[n\left(\phi-\Psi_{n}\right)\right]\right\}, \\
v_{n} & =\left\langle\cos \left[n\left(\phi-\Psi_{n}\right)\right]\right\rangle .
\end{aligned}
$$


here $y$ is rapidity, $p_{T}$ is particle transverse momentum, $\phi$ is the azimuth between the $\overrightarrow{p_{T}}$ and the participant event plane, and $\Psi_{n}$ is the azimuthal angle of the event plane of $n$-th flow component. The averaging in Equation (2) is performed over all hadrons in a single event and over all events. The first term in Equation (1) represents the isotropic flow, whereas the sum is related to the anisotropic flow. The first components of the latter are known as directed, $v_{1}$, elliptic, $v_{2}$, triangular, $v_{3}$, flow, and so forth. It appears that in the energy range between $\sqrt{s}=4 \mathrm{GeV}$ and $\sqrt{s}=10 \mathrm{GeV}$ directed flow of protons at midrapidity, $\left.v_{1}^{p}\right|_{y=0}$, changes its sign from "normal" (for definition, see below) to "antiflow", whereas the directed flows of mesons and antiprotons remain antiflow-oriented. Also, it is well-known that the directed flow of hadrons should drop and even vanish in the vicinity of first order deconfinement phase transition [7-9]. This circumstance explains the interest to the study of directed flow at beam energy scan (BES) program at RHIC, at CERN SPS, and at future accelerators NICA JINR and FAIR GSI.

In the present study we employ two microscopic transport models, ultra-relativistic quantum molecular dynamics (UrQMD) [10,11] and quark-gluon string model (QGSM) [12-14], to investigate heavy-ion collisions in the energy range $4 \leq \sqrt{s} \leq 19.6 \mathrm{GeV}$. Of particular interest is the models ability to reproduce the basic peculiarities in the development of the directed flow of identified hadrons. The paper is organized as follows. Primary features of both models are sketched in Section 2. Section 3 presents the results of our study of the energy-, mass-, and $y$-dependence of $v_{1}$ of different hadron species. Time evolution of the $v_{1}$ at midrapidity is compared to the directed flow of hadrons frozen out at different times. The influence of the mean-field potentials on the development of $v_{1}$ is also studied. Conclusions are drawn in Section 4.

\section{Similarities and Differences Between Microscopic Models}

Both UrQMD [10,11] and QGSM [12-14] are Monte Carlo event generators designed for description of relativistic $h h, h A$ and $\mathrm{A}+\mathrm{A}$ interactions. The multiparticle production takes place via formation and fragmentation of specific colored objects, strings, stretching uniformly between the quarks, diquarks, and their antistates. The string tension is about $\kappa \approx 1 \mathrm{GeV} / \mathrm{fm}$, and strings break into hadrons via the Schwinger-like mechanism of (di)quark-anti(di)quark formation. However, both mechanisms of the string formation and the string fragmentation in the models are different.

There are two possible methods of string excitation. UrQMD employs the longitudinal excitation of strings which is characteristic for all Lund-based string models [15]. Here the mass of the string arises from the momentum transfer, and the strings are stretching between the constituents belonging to the same hadron. Also, for hard collisions with the momentum transfer larger than $1.5 \mathrm{GeV} / \mathrm{c}$ UrQMD employs PYTHIA [16]. QGSM utilizes the color exchange mechanism [17], in which the constituents at the string ends belong to different hadrons. The variety of subprocesses in the latter case is much richer compared to the longitudinal excitation. For the string fragmentation process the string models utilize three possible schemes. The first scenario, suggested by the Lund group [15], implies that the string always splits into a sub-string and a particle on the mass shell at the end of the fragmenting string. This option is realized in UrQMD. In the second scheme the string splits into two sub-strings according to the area law [18]. The third option is provided by the Field-Feynman mechanism [19]. Here the string fragmentation takes place independently from both ends of the string. This scenario is employed in QGSM.

Both models utilize available experimental information, such as hadron cross sections, resonance widths and decay modes. For the description of hadron-nucleus and nucleus-nucleus collisions hadronic cascade is used. Particle propagation between the collisions is governed by Hamilton equations of motion. To obey the uncertainty principle, newly produced particles can interact only after the certain formation time. The Pauli principle is implemented by blocking the final state if the outgoing phase space is already occupied. 


\section{Main Results}

To investigate the basic features of the directed flow of pions and protons in microscopic models we opted for three bombarding energies, $\sqrt{s}=4 \mathrm{GeV}, 7.7 \mathrm{GeV}$ and $19 \mathrm{GeV}$, three systems of colliding nuclei, $\mathrm{Au}+\mathrm{Au}, \mathrm{Cu}+\mathrm{Cu}$ and $\mathrm{S}+\mathrm{S}$, and three centrality intervals, $\sigma / \sigma_{\text {geo }}=0-10 \%, 10-40 \%$ and $40-80 \%$. We consider directed flow of protons, $v_{1}^{p}(y)$, first. These distributions are displayed in Figure 1. According to definition [20,21], if the product of particle momentum along $x$-axis and rapidity is positive, $p_{x} \cdot y>0$, the flow is considered as "normal". In the opposite case, i.e., if $p_{x} \cdot y<0$, we call it "antiflow". Note that at ultrarelativistic energies, the directed flow of both protons and pions at midrapidity is practically zero. Let us see how the directed flow of protons in $\mathrm{Au}+\mathrm{Au}$ collisions is changing with decreasing collision energy from $19 \mathrm{GeV}$ to $4 \mathrm{GeV}$. Firstly, calculations are done with the QGSM. For peripheral collisions $v_{1}^{p}(y)$ has a characteristic wiggle structure, see bottom plots in Figure 1. It demonstrates weak antiflow at midrapidity. For semicentral $\mathrm{Cu}+\mathrm{Cu}$ and $\mathrm{S}+\mathrm{S}$ collisions with centrality $0-10 \%$ one sees antiflow at $\sqrt{s}=19.6 \mathrm{GeV}$, however, at $\sqrt{s}=7.7 \mathrm{GeV}$ the directed flow of protons is already normally elongated. This transformation is happening because the flying away baryon-rich remnants of colliding nuclei are closer to midrapidity zone, and the directed flow of charged hadrons in the remnants is developed in normal direction. Moreover, transition from antiflow to normal flow occurs earlier (in energy scale) in heavy-ion collisions compared to the light-ion ones. The explanation of this effect is as follows [22-25]. Hadrons are produced more copiously in heavy-ion collisions than in light-ion collisions at the same centrality range. Hadrons emitted in the direction of nuclear remnants will interact further thus acquiring an extra momentum. These hadrons will be pushed from the midrapidity area to higher rapidity regions. For a light-ion system with lower multiplicity of secondary hadrons the loss of a few hadrons emitted in normal flow direction would be more noticeable compared to the heavy-ion system. It is worth noting that this effect is opposite to the reduction (or softening) of directed flow caused by the quark-gluon plasma (QGP) formation. The QGP is expected to be produced in (semi)central heavy-ion collisions rather than in light-ion ones. Therefore, the softening of proton directed flow at $y=0$ should set in earlier in $\mathrm{Au}+\mathrm{Au}$ collisions compared to $\mathrm{Cu}+\mathrm{Cu}$ or $\mathrm{S}+\mathrm{S}$ collisions at the same energy.

For pions the picture is more permanent, as shown in Figure 2. Here the directed flow of pions at midrapidity demonstrates a distinct antiflow behavior for both heavy- and light-ion colliding systems, at all three bombarding energies, and for all three centrality bins.

Similar behavior is also observed in UrQMD calculations. To see the change of the proton flow direction clearly we present in Figure $3 v_{1}^{p}(y)$ for $\mathrm{S}+\mathrm{S}$ collisions at $\sqrt{s}=3.5 \mathrm{GeV}, 7.7 \mathrm{GeV}$ and $11.6 \mathrm{GeV}$, respectively. At lower collision energies the peaks, associated with the proton flow in the nuclei remnants areas, become closer to the midrapidity zone. Thus, protons with normal flow also start to determine the directed flow of protons at midrapidity. Directed flow of pions in these reactions is shown in Figure 4. It demonstrates a clear antiflow, which is slightly increasing with decreasing bombarding energy.

These features of the hadronic flow definitely need further investigation. Figure 5 (upper row) presents the snapshot of particle directed flows and densities for protons, antiprotons and charged pions in $\mathrm{Au}+\mathrm{Au}$ collisions with the impact parameter $b=6 \mathrm{fm}$ at $\sqrt{s}=7.7 \mathrm{GeV}$ after $10 \mathrm{fm} / \mathrm{c}$ of beginning of the collision. The whole space was subdivided into cells with volume $V=3 \mathrm{fm}^{3}$. The arrows indicate the collective velocities calculated for each cell. Density contours show that spatial distributions of $p, \bar{p}$ and $\pi^{ \pm}$are quite different. Protons are strongly influenced by the spectators, whereas the distributions of pions and antiprotons are more symmetric w.r.t. $z=0$. The arising directed flow is a result of superposition of the partial flows in the cells, each having either positive (normal flow) or negative (antiflow) sign. Evolution of the directed flow of these three particle species at midrapidity is displayed in Figure 5 (bottom row). Although both normal flow and antiflow of all species are quickly developed within the first $1.5 \mathrm{fm} / \mathrm{c}$, their resulting flow is weak compared to both flow and antiflow, components. It looks like the development of directed flow takes time longer than $t=14 \mathrm{fm} / \mathrm{c}$ even at midrapidity. 


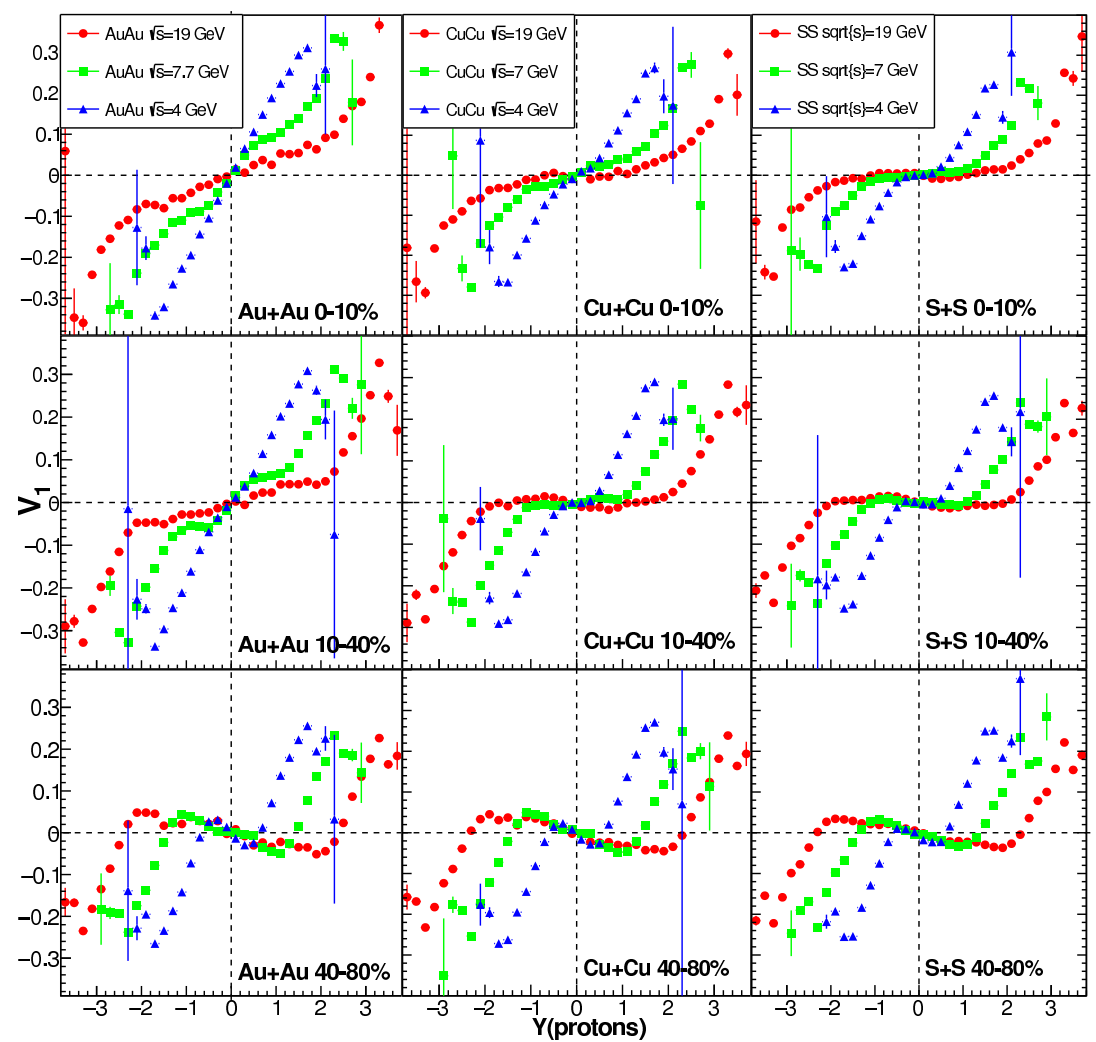

Figure 1. Directed flow of protons vs rapidity in QGSM calculations of $\mathrm{Au}+\mathrm{Au}$ (left column), $\mathrm{Cu}+\mathrm{Cu}$ (middle column) and S+S (right column) collisions at $\sqrt{s}=4 \mathrm{GeV}$ (blue points), $7.7 \mathrm{GeV}$ (green points) and $19 \mathrm{GeV}$ (red points) with centrality 0-10\% (upper row), 10-40\% (middle row) and 40-80\% (bottom row), respectively.

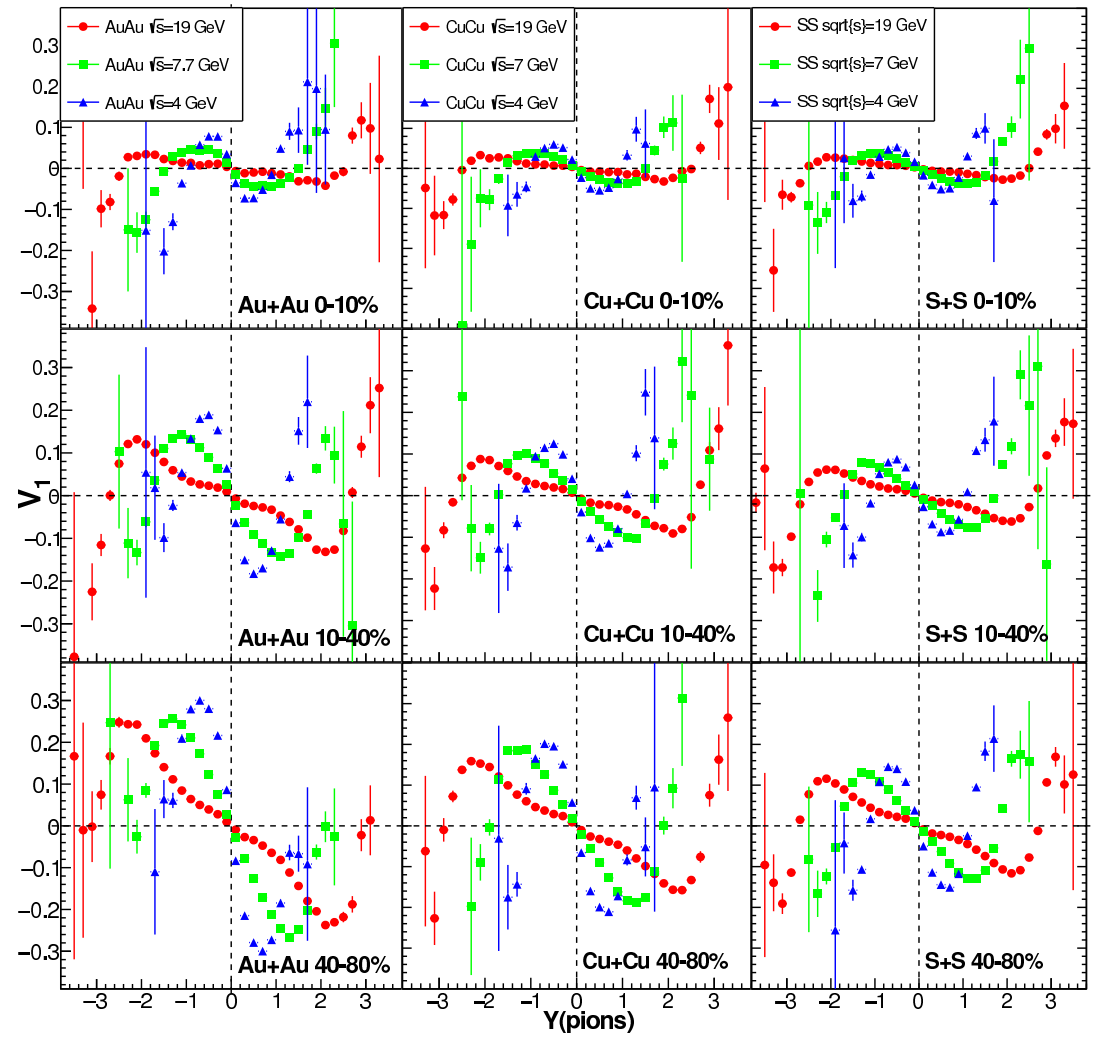

Figure 2. The same as Figure 1 but for the directed flow of $\pi^{ \pm}$. 


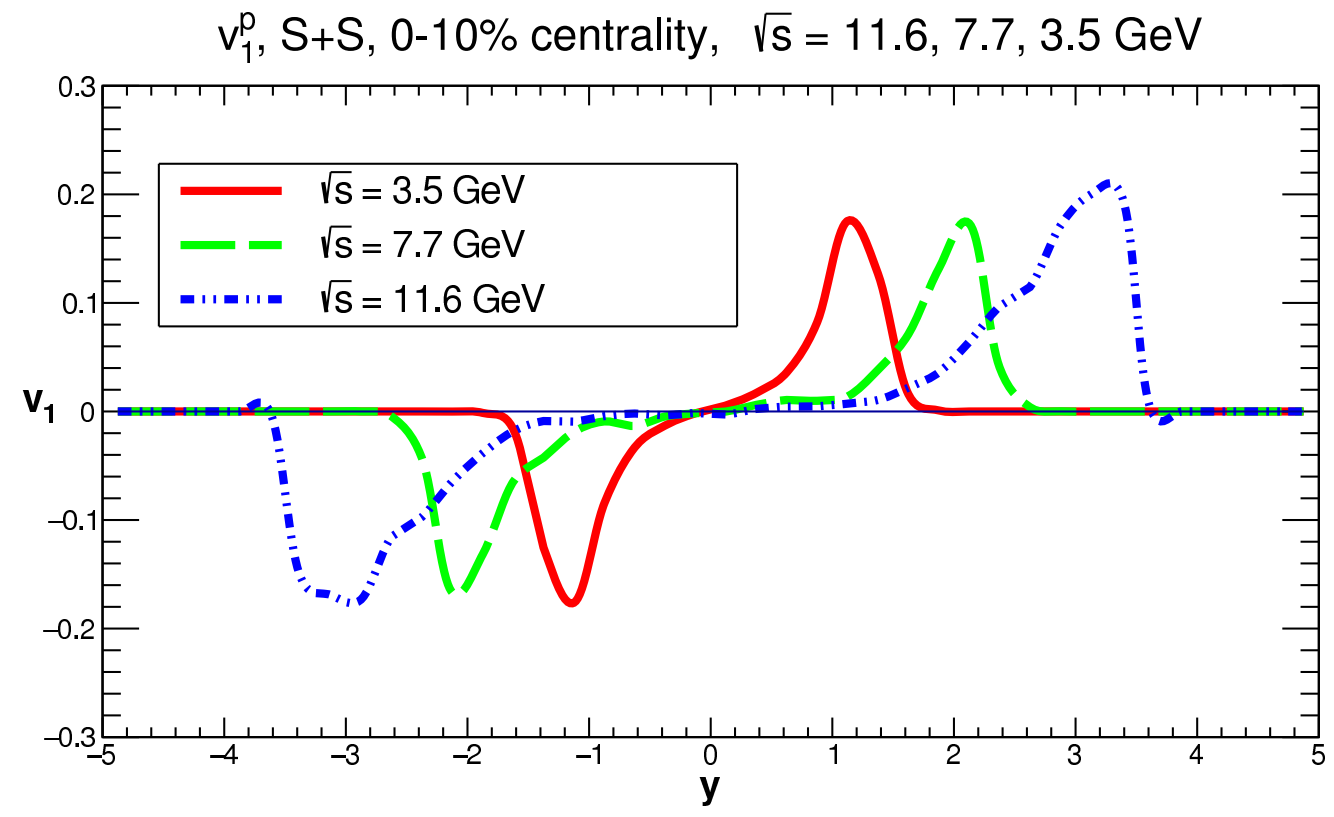

Figure 3. Directed flow of protons as a function of rapidity in UrQMD calculations of S+S collisions with centrality $0-10 \%$ at $\sqrt{s}=3.5 \mathrm{GeV}$ (solid line), $7.7 \mathrm{GeV}$ (dashed line), and $11.6 \mathrm{GeV}$ (dash-dotted line), respectively.

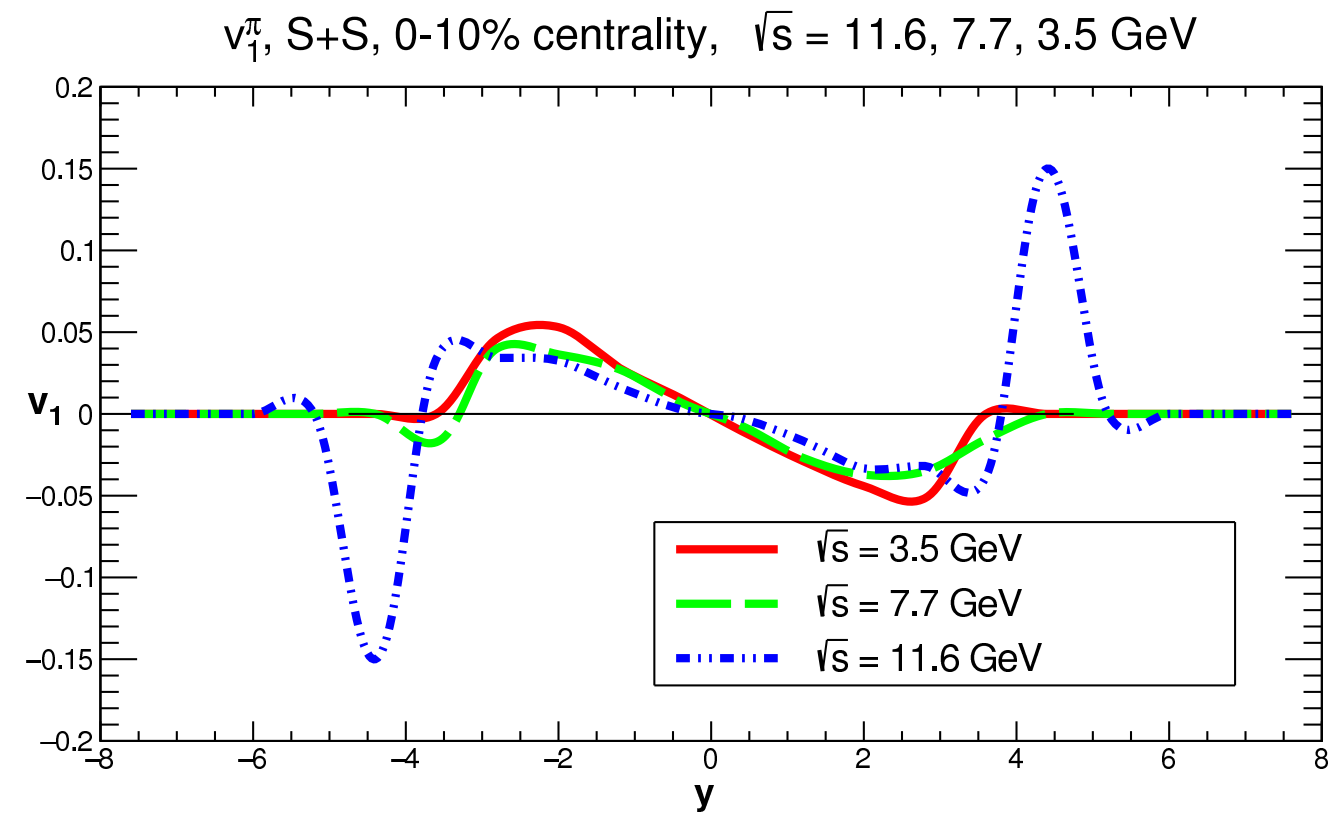

Figure 4. The same as Figure 3 but for the directed flow of $\pi^{ \pm}$.

Our next investigation concerns the evolution of partial differential flows of protons, charged pions and kaons, and Lambdas at $y=0$. Results of the calculations for Au+Au collisions with $b=6 \mathrm{fm}$ at $\sqrt{s}=11.6 \mathrm{GeV}$ are shown in Figure 6. Each plot displays two distributions. The first distribution, labeled as "time slices", presents the partial $v_{1}$ of a certain hadron species at midrapidity taken from $t=2 \mathrm{fm} / \mathrm{c}$ with the time step $1 \mathrm{fm} / \mathrm{c}$, i.e., at $t=2 \mathrm{fm} / \mathrm{c}, 3 \mathrm{fm} / \mathrm{c}, 4 \mathrm{fm} / \mathrm{c}$ and so forth. However, not all of these hadrons contribute to final midrapidity flow after the freeze-out of particles. The second distribution, therefore, represents the $\left.v_{1}\right|_{y=0}$ of hadrons from the final spectrum frozen out at $t=2.5 \pm 0.5 \mathrm{fm} / \mathrm{c}, 3.5 \pm 0.5 \mathrm{fm} / \mathrm{c}$, etc. For all particle species the two curves converge to each other at $12 \leq t \leq 15 \mathrm{fm} / \mathrm{c}$. 
$A u+A u, \sqrt{s}=7.7 \mathrm{GeV}, b=6 \mathrm{fm}, \mathrm{t}=10 \mathrm{fm} / \mathrm{c}, \mathrm{UrQMD}-3.4$
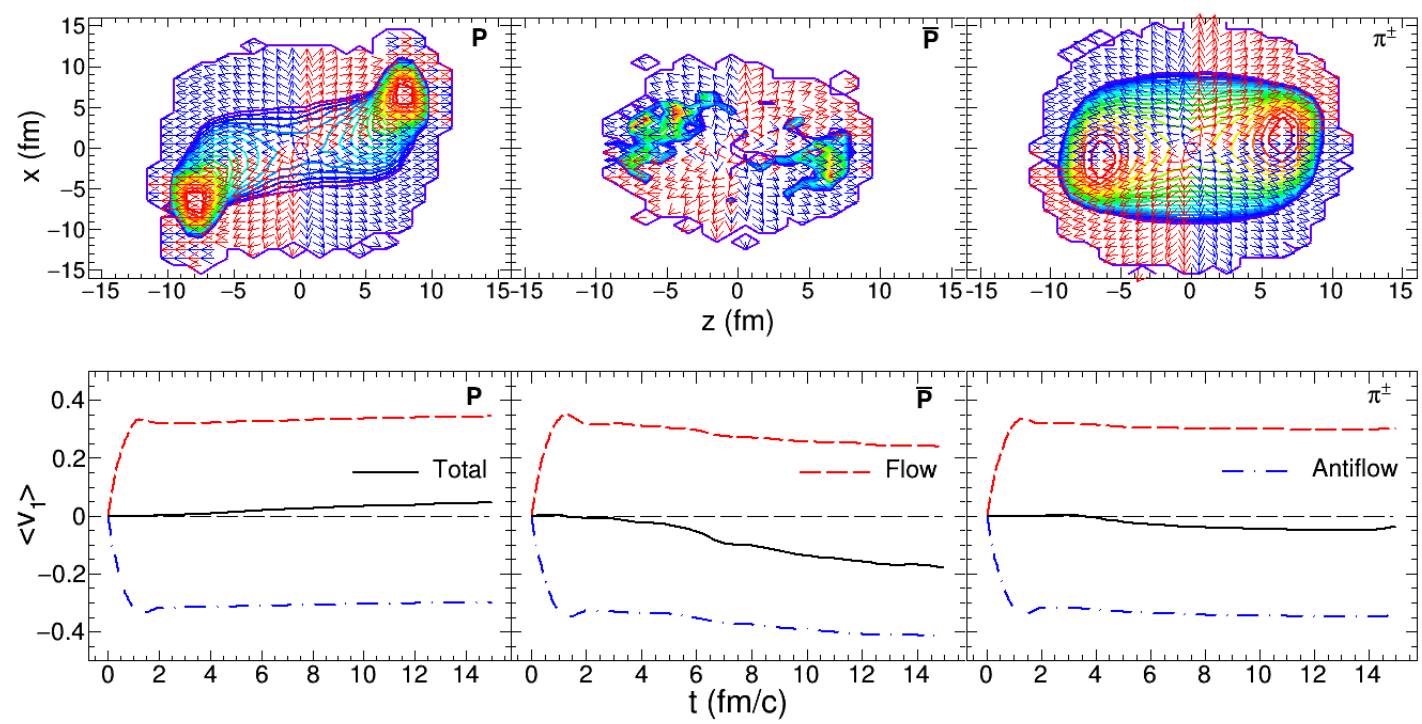

Figure 5. Upper row: The snapshot at time $t=10 \mathrm{fm} / \mathrm{c}$ of hadron densities (contour plots) and collective velocities (arrows) of the cells, each with volume $V=3 \mathrm{fm}^{3}$, for protons (left), antiprotons (middle), and charged pions (right) in UrQMD calculations of Au+Au collisions at $\sqrt{s}=7.7 \mathrm{GeV}$ with $b=6 \mathrm{fm}$. Bottom row: The time development of total directed flow (solid line) and partial flows in normal flow (red dashed line) and antiflow (blue dash-dotted line) directions of protons (left), antiprotons (middle), and charged pions (right) in these reactions.

One can see from this comparison that baryons, which are decoupled from the system at times $4 \leq t \leq 8 \mathrm{fm} / \mathrm{c}$, carry quite strong directed flow at midrapidity. However, if we would stop all interactions between the hadrons at this moment, the flow developed by the baryons will be weak. It is also weaker than the final directed flow of baryons after the particle freeze-out. For mesons the picture is similar to that with baryons but in terms of the antiflow. Both the pions and kaons emitted earlier carry stronger antiflow at midrapidity, whereas the $\left.v_{1}\right|_{y=0}$ of these particles is slowly increasing up to quite late times.

$A u+A u, \sqrt{s}=11.6 \mathrm{GeV}, \mathrm{b}=6 \mathrm{fm}, \mathrm{UrQMD}-3.4$

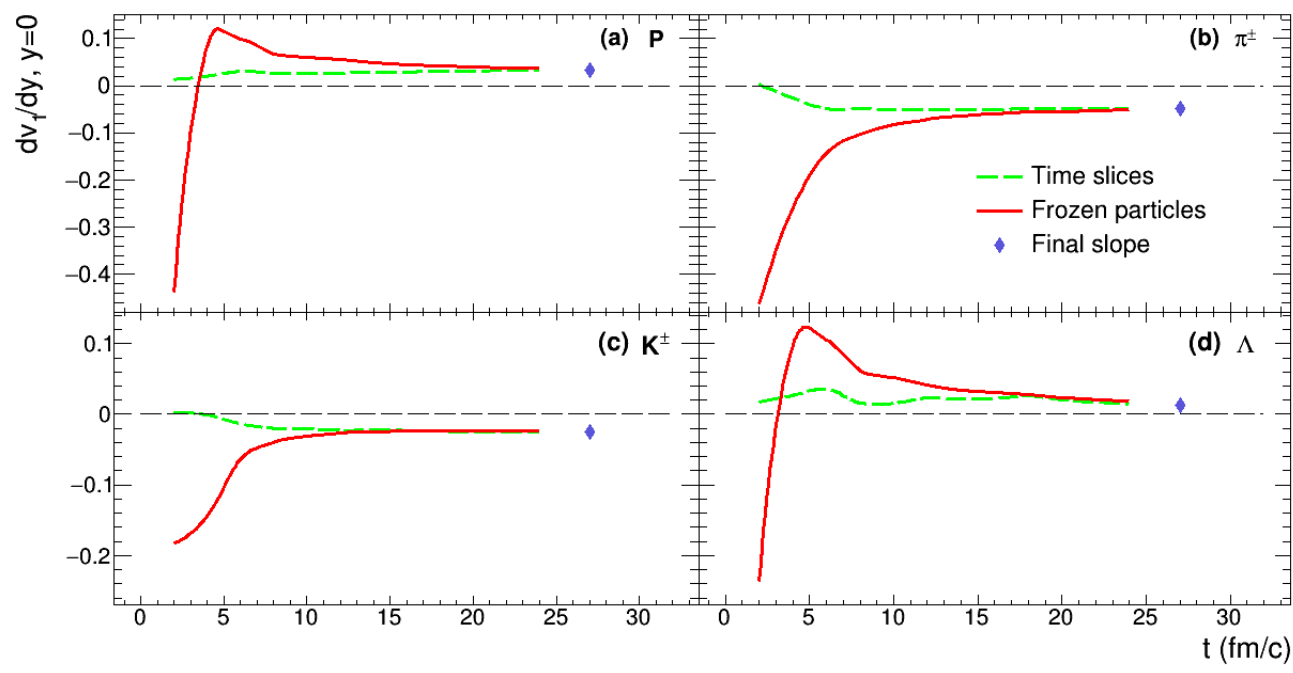

Figure 6. Time evolution of directed flow (green dashed curves) of $p, \pi^{ \pm}, K^{ \pm}$, and $\Lambda$ at midrapidity in UrQMD calculations of Au+Au collisions with $b=6 \mathrm{fm}$ at $\sqrt{s}=11.6 \mathrm{GeV}$. Directed flow carried by hadrons already frozen at a certain time is shown by red solid curves. Dots represent the final slopes of the hadron directed flows at midrapidity after the end of A+A collisions. 
The next problem is the investigation of the influence of mean fields on the strength of directed flow of identified hadrons. Recall that the mean-field potential in UrQMD consists of three parts [10], namely, Yukawa potential, Coulomb potential and Skyrme potential:

$$
V=V_{0}^{\gamma u k} \frac{e^{-\left|\vec{r}_{i}-\vec{r}_{j}\right| / \gamma_{Y}}}{\left|\vec{r}_{i}-\vec{r}_{j}\right|}+\frac{Z_{i} Z_{j} e^{2}}{\left|\vec{r}_{i}-\vec{r}_{j}\right|}+t_{1} \rho_{j}^{i n t}+t_{\gamma}(\gamma+1)^{-3 / 2}\left(\rho_{j}^{i n t}\right)^{\gamma},
$$

where

$$
\rho_{j}^{i n t}=\left(\frac{\alpha}{\pi}\right)^{3 / 2} e^{-\alpha r_{j}^{2}}
$$

Parameters of the hard and the soft potentials used in the calculations are listed in Table 1.

Table 1. Mean-field potential parameters.

\begin{tabular}{lcc}
\hline Parameter & Hard Potential (EoS1) & Soft Potential (EoS2) \\
\hline$\alpha\left(\mathrm{fm}^{-2}\right)$ & 0.25 & 0.25 \\
$t_{1}\left(\mathrm{MeV} \mathrm{fm}^{3}\right)$ & -163 & -353 \\
$t_{\gamma}\left(\mathrm{MeV} \mathrm{fm}^{6}\right)$ & 125.93 & 304 \\
$\gamma$ & 1.676 & 1.167 \\
$V_{0}^{Y u k}(\mathrm{MeV} \mathrm{fm})$ & -0.498 & 1.0038 \\
$\gamma^{Y u k}(\mathrm{fm})$ & 1.4 & 1.4 \\
\hline
\end{tabular}

Note that direct comparison of results of model calculations with the experimental data is a very non-trivial task. One has to know not only the (pseudo)rapidity and transverse momentum cuts, but also binning of $v_{1}(y)$ distributions and rapidity interval chosen for the fit, determine the centrality according the multiplicity distribution, etc. Thus, the main goal of our present paper is to study the general trends and present a model description of hadron directed flow rather than fine tuning the free parameters of the models. To study the influence of hard and soft mean field potentials on the directed flow of identified hadrons and energy dependence of the flow, we generated one million minimum bias $\mathrm{Au}+\mathrm{Au}$ collisions at center-of-mass energies $\sqrt{s}=4,7.7,11.5,19.6,32,62.4$ and $200 \mathrm{GeV}$, respectively. For each energy below $\sqrt{s}=19.6 \mathrm{GeV}$ one run was with the hard potential, another was with the soft potential, and the third one was without the mean fields. Multiplicity-based centrality separation was performed and semicentral (10-40\%) events were chosen for further analysis. The midrapidity slope of directed flow was determined within the interval $|y| \leq 0.5$. Because the position of the event plane (EP) in the experiment is unknown, we employed also the experimental procedure [26] of the event plane restoration to estimate the possible systematic errors. Results obtained for baryons, namely protons, antiprotons, Lambdas and antiLambdas, are shown in Figure 7. For comparison, experimental data of the STAR collaboration [26,27] are also plotted onto the UrQMD calculations. Calculated values of $d v_{1} /\left.d y\right|_{y=0}$ as a function of $\sqrt{s}$ for charged pions and charged kaons are displayed in Figure 8. One can see in Figure 7 that for protons and Lambdas the version without the mean fields provide fair quantitative description of the data. Calculations with hard potential (stiff equation of state, EOS1) provide too strong flow and antiflow of these particles, whereas the soft potential (EOS2) makes the flow weaker. For antibaryons, the soft potential provides better quantitative agreement with the experiment at $\sqrt{s}=11.5 \mathrm{GeV}$ but cannot match the data at $\sqrt{s}=7.7 \mathrm{GeV}$. Nevertheless, the changing of the sign from normal flow to antiflow for baryons within the interval $7.7 \leq \sqrt{s} \leq 11.5 \mathrm{GeV}$ and decrease of the antibaryon antiflow is reproduced. For charged mesons, both hard and soft potentials do not play a decisive role at $\sqrt{s} \geq 11.5 \mathrm{GeV}$. At lower energies all calculations predict stronger antiflow for all mesons except positive kaons. This interesting problem needs further investigation. 


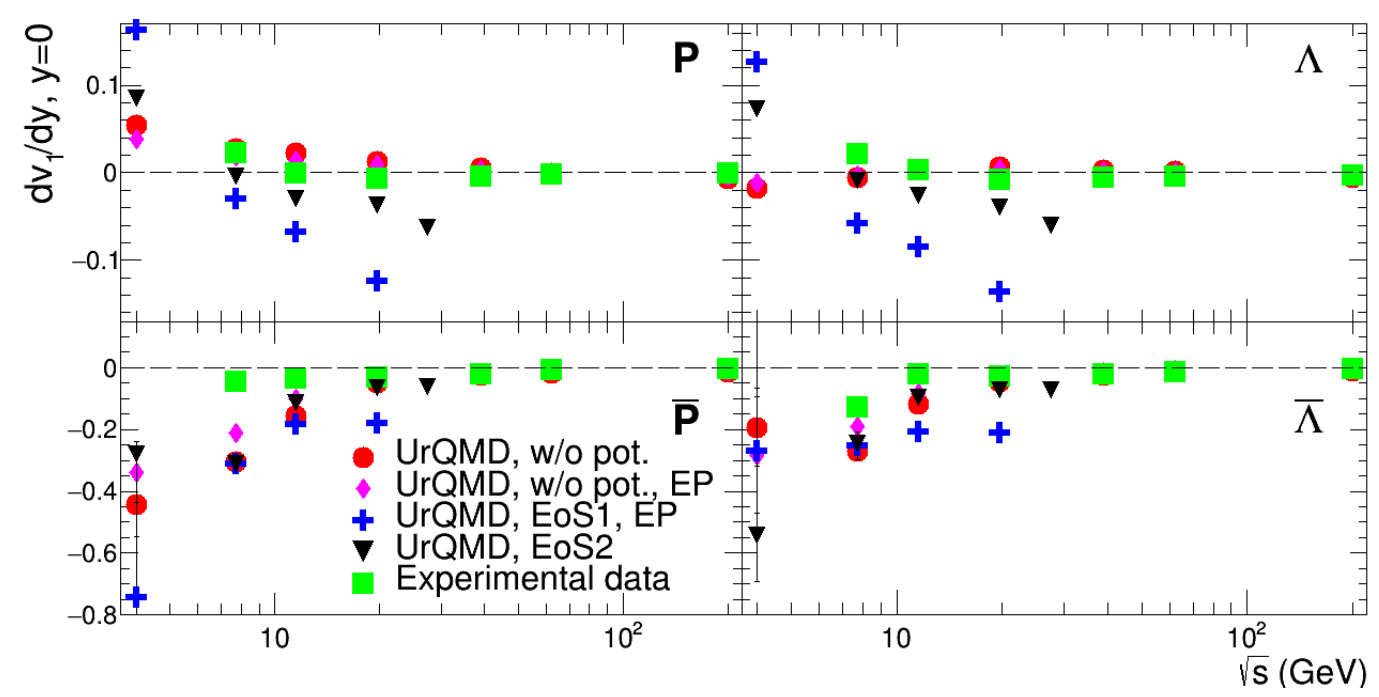

Figure 7. The slope of directed flow of $p, \bar{p}, \Lambda$, and $\bar{\Lambda}$ at $y=0$ as a function of $\sqrt{s}$ in UrQMD calculations with hard (crosses) and soft (diamonds) mean-field potentials, and without the mean fields (circles) of $\mathrm{Au}+\mathrm{Au}$ collisions with centrality 10-40\%. Squares denote the experimental data from [26,27].

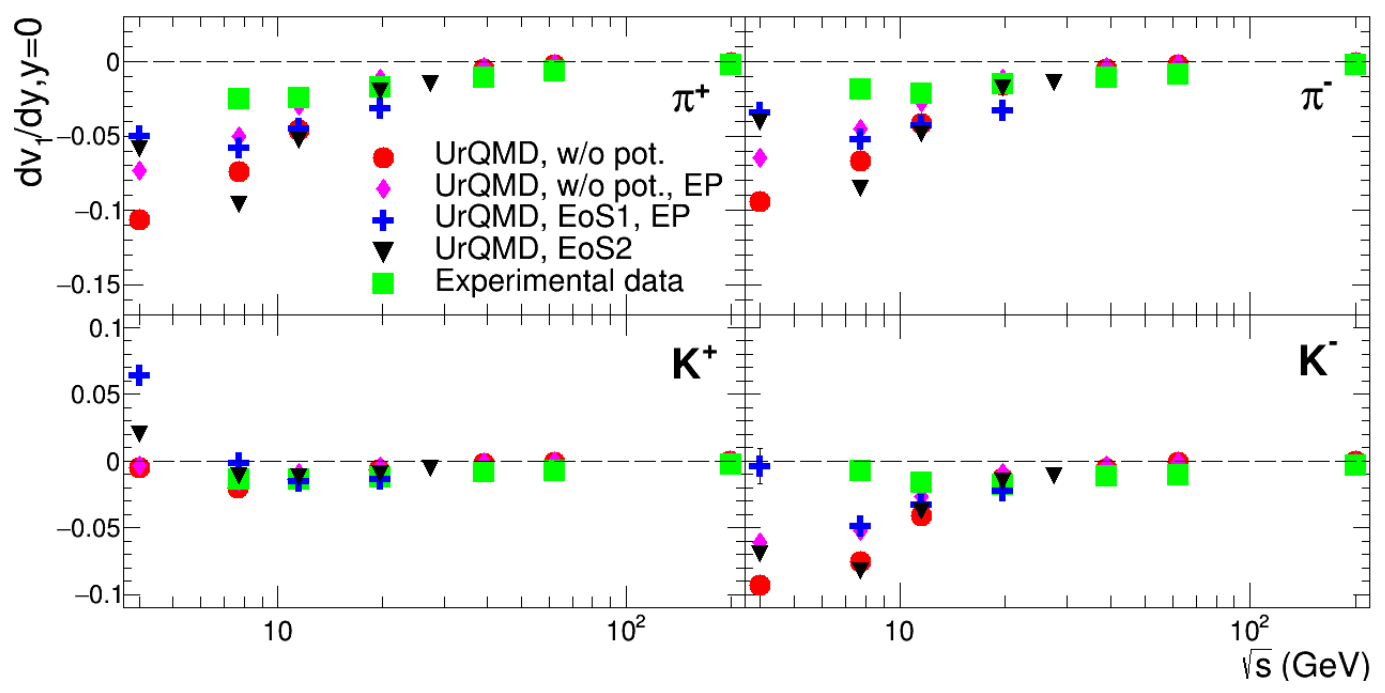

Figure 8. The same as Figure 7 but for the directed flow of $\pi^{+}, \pi^{-}, K^{+}$, and $K^{-}$, respectively.

\section{Conclusions}

Two microscopic transport models, UrQMD and QGSM, were used to study general features of directed flow of identified hadrons in heavy-ion collisions in the energy range $4 \mathrm{GeV} \leq \sqrt{s} \leq 19.6 \mathrm{GeV}$. Although the mechanisms of string excitation and string fragmentation in the models are different, both UrQMD and QGSM indicate that directed flow of protons and Lambdas is positive (normal flow) in central and semicentral collisions at $\sqrt{s} \leq 7.7 \mathrm{GeV}$. It is reduced and develops an antiflow with increasing bombarding energy and as the reaction becomes more peripheral. Moreover, this effect is stronger in light-ion collisions with the same centrality. This feature can be explained by the influence of dense baryon-rich remnants of colliding nuclei. Directed flows of antibaryons and mesons demonstrate stable weak antiflow behavior for all energies and all centralities.

Time evolution of partial directed flows of identified hadrons at midrapidity takes about $10-15 \mathrm{fm} / \mathrm{c}$ at collision energies around $11.6 \mathrm{GeV}$. These distributions can be decomposed onto two parts representing normal flow and antiflow. Both parts have quite substantial magnitudes, whereas the 
resulting flow is relatively weak. Calculations with hard and soft mean-field potentials of $\mathrm{Au}+\mathrm{Au}$ collisions at $\sqrt{s} \leq 19.6 \mathrm{GeV}$ show that these potentials influence mainly directed flow of baryons rather than that of mesons. The model calculations qualitatively reproduce the experimentally-observed trends. Quantitative description of the data in the intermediate energy range, however, is a complex problem demanding further investigation.

Author Contributions: All authors contributed equally to this work.

Funding: The work was supported by the Norwegian Centre for International Cooperation in Education (SIU) under grants "CPEA-LT-2016/10094-From Strong Interacting Matter to Dark Matter" and "UTF-2016-long-term/10076-Training of Bachelor, Master and PhD Students specialized in high energy physics". The work of L.V.B. and E.E.Z. was supported by the Norwegian Research Council (NFR) under grant No. 255253/F50 - "CERN Heavy Ion Theory". This work was also performed within the European network COST Action CA15213 "Theory of hot matter and relativistic heavy-ion collisions" (THOR).

Acknowledgments: Fruitful discussions with L. Csernai and Yu. Ivanov are gratefully acknowledged.

Conflicts of Interest: The authors declare no conflict of interests.

\section{References}

1. Scheid, W.; Müller, H.; Greiner, W. Nuclear shock waves in heavy-ion collisions. Phys. Rev. Lett. 1974, 32, 741-744. [CrossRef]

2. Stöcker, H.; Maruhn, J.A.; Greiner, W. Collective sideward flow of nuclear matter in violent high-energy heavy ion collisions. Phys. Rev. Lett. 1980, 44, 725-728. [CrossRef]

3. Gustafsson, H.A.; Gutbrod, H.H.; Kolb, B.; Löhner, H.; Ludewigt, B.; Poskanzer, A.M.; Renner, T.; Riedesel, H.; Ritter, H.G.; Warwick, A.; et al. Collective flow observed in relativistic nuclear collisions. Phys. Rev. Lett. 1984, 52, 1590-1593. [CrossRef]

4. Reisdorf, W.; Ritter, H.G. Collective flow in heavy-ion collisions. Ann. Rev. Nucl. Part. Sci. 1997, 47, 663-709. [CrossRef]

5. Voloshin, S.; Zhang, Y.; Flow study in relativistic nuclear collisions by Fourier expansion of azimuthal particle distributions. Z. Phys. C 1996, 70, 665-672. [CrossRef]

6. Poskanzer, A.M.; Voloshin, S.A. Methods for analyzing anisotropic flow in relativistic nuclear collisions. Phys. Rev. C 1998, 58, 1671-1678. [CrossRef]

7. Hung, C.M.; Shuryak, E.V. Hydrodynamics near the QCD phase transition: Looking for the longest lived fireball. Phys. Rev. Lett. 1995, 75, 4003-4006. [CrossRef] [PubMed]

8. Rischke, D.H.; Gyulassy, M. The time delay signature of quark-gluon plasma formation in relativistic nuclear collisions. Nucl. Phys. A 1996, 608, 479-512. [CrossRef]

9. Ivanov, Y.B.; Soldatov, A.A. Directed flow indicates a cross-over deconfinement transition in relativistic nuclear collisions. Phys. Rev. C 2015, 91, 024915. [CrossRef]

10. Bass, S.A.; Belkacem, M.; Bleicher, M.; Brandstetter, M.; Bravina, L.; Ernst, C.; Gerland, L.; Hofmann, M.; Hofmann, S.; Konopka, J.; et al. Microscopic models for ultrarelativistic heavy ion collisions. Prog. Part. Nucl. Phys. 1998, 41, 255-369. [CrossRef]

11. Bleicher, M.; Zabrodin, E.; Spieles, C.; Bass, S.A.; Ernst, C.; Soff, S.; Bravina, L.; Belkacem, M.; Weber, H.; Stöcker, H.; et al. Relativistic hadron hadron collisions in the ultrarelativistic quantum molecular dynamics model. J. Phys. G 1999, 25, 1859-1896. [CrossRef]

12. Amelin, N.S.; Bravina, L.V. The Monte Carlo Realization of Quark-Gluon String Model for Description of High-energy Hadron Hadron Interactions. Sov. J. Nucl. Phys. 1990, 51, 133-140.

13. Bravina, L.V.; Mishustin, I.N.; Bondorf, J.P.; Faessler, A.; Zabrodin, E.E. Microscopic study of freezeout in relativistic heavy ion collisions at SPS energies. Phys. Rev. C 1999, 60, 044905. [CrossRef]

14. Bleibel, J; Bravina, L.V.; Zabrodin, E.E. How many of the scaling trends in pp collisions will be violated at $\sqrt{s_{N N}}=14 \mathrm{TeV}$ ?-Predictions from Monte Carlo quark-gluon string model. Phys. Rev. D 2016, 93, 114012. [CrossRef]

15. Andersson, B.; Gustafson, G.; Nilsson-Almqvist, B. A Model for Low p(t) Hadronic Reactions, with Generalizations to Hadron-Nucleus and Nucleus-Nucleus Collisions. Nucl. Phys. B 1987, 281, 289-309. [CrossRef] 
16. Sjostrand, T.; Mrenna, S.; Skands, P. A Brief Introduction to PYTHIA 8.1. Comput. Phys. Commun. 2008, 178, 852-867. [CrossRef]

17. Kaidalov, A.B. Soft interactions of hadrons in QCD. Surveys in High Energy Phys. 1999, 13, 265-330. [CrossRef]

18. Artru, X.; Mennessier, G. String model and multiproduction. Nucl. Phys. B 1974, 70, 93-115. [CrossRef]

19. Field, R.D.; Feynman, R.P. A Parametrization of the Properties of Quark Jets. Nucl. Phys. B 1978, 136, 1-76. [CrossRef]

20. Bravina, L.V. Scaling violation of transverse flow in heavy ion collisions at AGS energies. Phys. Lett. B 1995, 344, 49-54. [CrossRef]

21. Brachmann, J.; Soff, S.; Dumitru, A.; Stoecker, H.; Maruhn, J.A.; Greiner, W.; Bravina, L.V.; Rischke, D.H. Antiflow of nucleons at the softest point of the EoS. Phys. Rev. C 2001, 61, 024909. [CrossRef]

22. Bravina, L.V.; Faessler, A.; Fuchs, C.; Zabrodin, E.E. Microscopic study of energy and centrality dependence of transverse collective flow in heavy ion collisions. Phys. Rev. C 2000, 61, 064902. [CrossRef]

23. Zabrodin, E.E.; Fuchs, C.; Bravina, L.V.; Faessler, A. Transverse momentum dependence of directed particle flow at 160A-GeV. Phys. Rev. C 2001, 63, 034902. [CrossRef]

24. Bravina, L.V.; Zabrodin, E.E. Directed flow in heavy-ion collisions at NICA: What is interesting to measure? Eur. Phys. J. A 2016, 52, 245. [CrossRef]

25. Bravina, L.V.; Kvasiuk, Y.; Sivoklokov, S.Y.; Vitiuk, O.; Zabrodin, E.E. Anisotropic (v1 and v2) flow in relativistic heavy-ion collisions at energies between $4 \mathrm{GeV}$ and $200 \mathrm{GeV}$. In Proceedings of the 20th International Seminar on High Energy Physics (Quarks 2018), Valday, Russia, 27 May-2 Jun 2018; EDP Sciences: Paris, France, 2018; Volume 191, p. 05004.

26. Adamczyk, L.; Adkins, J.K.; Agakishiev, G.; Aggarwal, M.M.; Ahammed, Z.; Alekseev, I.; Alford, J.; Anson, C.D.; Aparin, A.; Arkhipkin, D.; et al. (STAR Collaboration). Beam-Energy Dependence of the Directed Flow of Protons, Antiprotons, and Pions in Au+Au Collisions. Phys. Rev. Lett. 2014, 112, 162301. [CrossRef] [PubMed]

27. Adamczyk, L.; Adams, J.R.; Adkins, J.K.; Agakishiev, G.; Aggarwal, M.M.; Ahammed, Z.; Ajitanand, N.N.; Alekseev, I.; Anderson, D.M.; Aoyama, R.; et al. (STAR Collaboration). Beam-Energy Dependence of Directed Flow of $\Lambda, \bar{\Lambda}, K^{ \pm}, K_{s}^{0}$ and $\phi$ in Au+Au Collisions. Phys. Rev. Lett. 2018, 120, 062301. [CrossRef] [PubMed]

(C) 2019 by the authors. Licensee MDPI, Basel, Switzerland. This article is an open access article distributed under the terms and conditions of the Creative Commons Attribution (CC BY) license (http://creativecommons.org/licenses/by/4.0/). 